\title{
Performance Analysis of Co- and Cross-tier Device-to-Device Communication Underlaying Macro-small Cell Wireless Networks
}

\author{
Tong Li ${ }^{1,2}$, Zhu Xiao ${ }^{1,2}$, Hassana Maigary Georges ${ }^{1}$, Zhinian Luo ${ }^{1}$, Dong Wang ${ }^{1}$ \\ ${ }^{1}$ College of Computer Science and Electronic Engineering, Hunan University \\ Changsha, China \\ ${ }^{2}$ State Key Laboratory of Integrated Services Networks, Xidian University \\ Xi'an, China \\ [e-mail: \{litong, zhxiao, zhinianluo,wangd\}@hnu.edu.cn, hamjorg@yahoo.fr] \\ *Corresponding author: Zhu Xiao
}

Received November 29, 2015; revised February 5, 2016; accepted March 6, 2016; published April 30, 2016

\begin{abstract}
Device-to-Device (D2D) communication underlaying macro-small cell networks, as one of the promising technologies in the era of 5G, is able to improve spectral efficiency and increase system capacity. In this paper, we model the cross- and co-tier D2D communications in two-tier macro-small cell networks. To avoid the complicated interference for cross-tier D2D, we propose a mode selection scheme with a dedicated resource sharing strategy. For co-tier D2D, we formulate a joint optimization problem of power control and resource reuse with the aim of maximizing the overall outage capacity. To solve this non-convex optimization problem, we devise a heuristic algorithm to obtain a suboptimal solution and reduce the computational complexity. System-level simulations demonstrate the effectiveness of the proposed method, which can provide enhanced system performance and guarantee the quality-of-service (QoS) of all devices in two-tier macro-small cell networks. In addition, our study reveals the high potential of introducing cross- and co-tier D2D in small cell networks: $i$ ) cross-tier D2D obtains better performance at low and medium small cell densities than co-tier D2D, and ii) co-tier D2D achieves a steady performance improvement with the increase of small cell density.
\end{abstract}

Keywords: Device-to-Device, small cell, interference management, two-tier networks, performance evaluation 


\section{Introduction}

$\mathbf{W}_{\text {ith the exponential increase of mobile data demand, heterogeneous networks, which }}$ combine different wireless access technologies, standards, and protocols, are considered the basic architecture of future 5G cellular networks [1]. Moreover, in the age of data, it will be more common for several co-located devices to want to share content wirelessly [2]. Therefore, Device-to-Device (D2D) communication, which allows devices to communicate directly under the control of a base station (BS), is becoming a promising heterogeneous technology to help 5G cellular communication networks cope with the explosive increase of data traffic [3] [4].

In 2010, Qualcomm pioneered a mobile communication system, FlashLinQ, which implements wireless sensing to enable proximity-aware communication among devices [5]. In addition, the Third Generation Partnership Project (3GPP) has been investigating D2D communications as Proximity Services (ProSe), which mainly include two parts, ProSe discovery and ProSe communication. Furthermore, they have defined ProSe-enabled user equipment as equipment that supports ProSe discovery and ProSe communication. In particular, the feasibility of ProSe and its use-cases in LTE are studied in [6], and the channel models for LTE ProSe and physical layer options are defined in [7].

In a D2D communication system, each D2D pair has two basic communication modes, cellular mode (CM) and direct communication mode (i.e., D2D mode) [8]. In the direct mode, data are transmitted to the receiver directly, while the cellular mode requires a base station to relay.

Unlike wireless local-area network technologies (e.g., Wi-Fi, Wi-Fi Direct) and wireless personal-area network technologies (e.g., Bluetooth, ZigBee), D2D communication users do not need to manually pair with their peers. Instead, the D2D communication procedure is invisible for users because the BS handles the peer discovery, D2D pairing and mode selection entirely [9].

\subsection{Related Work}

D2D communication can be divided into two categories based on the spectrum in which D2D communication occurs [10]: inband D2D communication and outband D2D communication. The communication under the inband category occurs on the licensed spectrum (i.e., the cellular spectrum), which is used for both D2D and cellular users. The D2D users in the outband category exploit the unlicensed spectrum. Because the interference in the unlicensed spectrum is hard to manage and the QoS of users is difficult to guarantee, researchers mainly focus on inband D2D communication, which can be fully controlled by BSs [11].

In an inband D2D communication system, D2D pairs under the direct communication mode may use cellular spectrum resources in either an orthogonal or nonorthogonal manner. In the nonorthogonal case, inter-channel interference may occur between cellular users and D2D users. In the other case, the orthogonal case, D2D pairs use dedicated resources but cannot exploit the reuse potential of D2D communication to improve the spectral efficiency. Because inter-channel interference between cellular and D2D links exists and may severely deteriorate the overall system performance [12], interference management is one of the critical challenges and research aspects for D2D communication underlaying cellular networks [9][13][14][15][16][17]. 
The authors in [9] studied the case in which one cellular user and one D2D pair share the radio resources and aimed to optimize the overall cell throughput with resource allocation and power control strategies while giving priority to the cellular user. In [13], the authors considered D2D communication to share uplink cellular resources in a multi-user cellular system and analysed the globally optimal resource sharing strategy. The authors in [14] presented game-theoretic resource allocation methods for selfish D2D users underlying a cellular network. For the sake of mitigating the interference from cellular transmission to the D2D link, a distance-constrained resource-sharing criterion is proposed in [15]. [16] studied the resource sharing between two types of users, cellular users and D2D users, and regarded the channel allocation as a $0-1$ assignment problem that can be solved by the Hungarian algorithm. In addition, [17] considered the D2D communication underlaying cellular networks with a multi-antenna BS and proposed to change the precoding vector to mitigate or cancel the interference from BS to D2D users.

\subsection{Motivations and Contributions}

The future $5 \mathrm{G}$ cellular communication system is expected to be a mixed network of various technologies and devices [18]. The combination of D2D communication and cellular networks is an overwhelming trend [19]. Furthermore, the cellular network has developed as a multi-tier network that comprises a conventional cellular network (i.e., macrocell network) with multiple low-power base stations (i.e., small cells) [20]. Different network tiers share the same radio spectrum. Therefore, interference management will be a key research challenge with the co-existence of D2D communications and multi-tier cellular networks because the interference situation is more complicated [21][22].

The existing works focus on D2D communications in a single-tier network. The authors in [9][17] considered the case in which only one cellular user and two D2D users (i.e., one D2D pair) co-exist in one BS network. [13] and [15] studied the scenario in which one D2D pair share uplink resources in a cellular system consisting of one BS and multiple cellular users. In [16], the authors studied a single cell environment where multiple D2D pairs and multiple cellular users are co-deployed. There are some investigations of D2D communication in multi-tier networks as well [23][24]. The authors in [23] obtained the per user average rate within stochastic geometry analysis for a D2D overlaying two-tier cellular network in which the different network tiers are allocated separated spectrum. [24] proposed an auction-based distributed solution to allocate radio resources in a D2D-enabled multi-tier heterogeneous network, but only considered the case in which D2D pairs are all in macrocell tier.

In this paper, we study the D2D communications underlaying co-channel deployed macro-small cell networks that are composed of two clearly separated tiers, the macrocell tier and the small cell tier. We focus on the performance of the co- and cross-tier D2D communication in such two-tier networks by jointly considering mode selection, power control and resource reuse strategies. Co-tier D2D means both of the devices in a D2D pair are with the same network tier. Cross-tier D2D means the D2D pair devices belong to different network tiers, for example, one user is with the small cell tier and the other is with the macrocell tier.

The contributions of our work are summarized as follows.

- For the two-tier network architecture, we propose two resource-sharing strategies, the cross-tier resource sharing strategy (CTSS) and dedicated resource sharing strategy (DS) for co- and cross-tier D2D users, respectively, to avoid interference between D2D users and cellular users in the same tier. 
- We formulate the joint optimization problem of power control and resource reuse for co-tier D2D with the objective of maximizing the overall outage capacity considering QoS constraints of cellular and D2D users. To solve this non-convex combinatorial optimization problem, we propose a heuristic algorithm to tackle the high computational complexity.

The rest of the paper is organized as follows. In section 2, we describe the co- and cross-tier D2D communications underlaying macro-small cell networks. In section 3, we present the interference issue in the system and formulate it as a non-convex combinatorial optimization problem. In section 4, we devise a suboptimal heuristic algorithm with joint power control and resource reuse for co-tier D2D. In section 5, we present extensive simulation to confirm the effectiveness of the proposed algorithm. Section 6 concludes this paper.

\section{Cross- and Co-tier D2D Underlaying Macro-small Cell Networks}

\subsection{System Model}

In this paper, we focus on macro-small cell networks enabling co-tier and cross-tier D2D communications. In such two-tier networks, the mobile users consist of cellular users and D2D users, which can be with either the macrocell tier or small cell tier. We divide the mobile users into four categories according to their communication methods (i.e., cellular or D2D communication) and their network tiers (i.e., the macrocell tier or small cell tier), as depicted in Fig. 1.

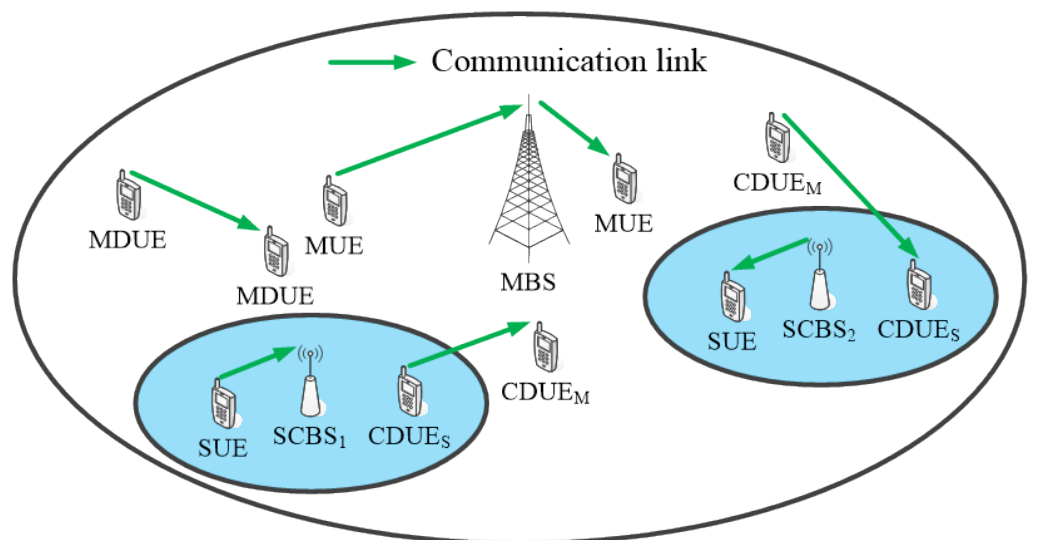

Fig. 1. Various types of mobile users in macro-small cell networks enabling D2D communication.

- Macrocell cellular user equipment (MUE): The devices with the cellular mode in a macrocell include cellular users and the D2D users using cellular mode (CM). This type of user is associated with the macrocell.

- Macrocell D2D user equipment (MDUE): Both of the D2D pair users belong to the macrocell tier, and the D2D pair uses the D2D mode to communicate directly, i.e., the co-tier D2D considered in this study. This type of user is associated with the macrocell as well.

- Small cell cellular user equipment (SUE): The devices with the cellular mode in a small cell include cellular users and the D2D users using cellular mode. This type of user is associated with the small cell.

- Cross-tier D2D user equipment (CDUE): The users of one D2D pair belong to different network tiers. Furthermore, the D2D pair uses D2D mode to communicate directly. As 
shown in Fig. 1, CDUE $E_{S}$ and $\mathrm{CDUE}_{\mathrm{M}}$ form a cross-tier D2D pair, in which $\mathrm{CDUE}_{\mathrm{S}}$ denotes the one within the coverage of a small cell and $C D U E_{M}$ denotes the other one in the coverage of the macrocell. It is noteworthy that $\mathrm{CDUE}_{\mathrm{S}}$ is associated with the small cell while $\mathrm{CDUE}_{\mathrm{M}}$ is associated with the macrocell.

For one D2D pair, whether it should be a CDUE or MDUE depends on its associated network tier. If both of the D2D pair users are associated with the macrocell tier (i.e., within the coverage of the macrocell), the D2D pair is a MDUE. Otherwise, if the D2D pair users are associated with different tiers, i.e., one user is within the coverage of a small cell while the other one is within the coverage of the macrocell, the D2D pair is a CDUE. That is caused by the two-tier network architecture.

We assumed that there are $N$ uplink resource blocks and $N$ downlink resource blocks (RBs) in the entire system, which are shared for both the macrocell and small cells, and all users are ProSe-enabled user equipment, which is defined in [6]. We study the fully loaded case, i.e., there is a total of $2 N$ active users in the macrocell tier, $N$ uplink users and $N$ downlink users. There are $K$ small cells in the system. Let $2 M$ denote the number of users that belong to each small cell, and there are $M$ uplink users and $M$ downlink users. It is noteworthy that each macrocell user is only allowed to occupy one RB, while each small cell user can use multiple RBs. That is because macrocell BSs are designed for the coverage of large areas and are capable of basic communication services, while small cells are designed to realize the high data demand in hotspots [25].

In the cellular networks, the forming of a D2D pair can be treated as a random event [11]. Let $P$ denote the probability that D2D communication takes place for each user, and $P_{C}$ and $P_{M}$ are the proportion of CDUE and MDUE to the entire set of D2D users, respectively; obviously, $P_{C}+P_{M}=1$. Therefore, there are $\left\lfloor 2 N P P_{M}\right\rfloor$ macrocell D2D users (MDUE) in the macrocell tier and $\left\lfloor 2 N P P_{C}\right\rfloor$ cross-tier D2D users (CDUE) in the macrocell tier, where $\lfloor\cdot\rfloor$ is the trunc operation. Because the users of one MDUE pair both belong to the macrocell tier, the number of MDUE pairs equals $\left\lfloor 2 N P P_{M}\right\rfloor / 2=\left\lfloor N P P_{M}\right\rfloor$. The users of one CDUE pair belong to different network tiers, and one user is with the small cell tier while the other one is in the macrocell tier. Therefore, the number of CDUE pairs equals the number of cross-tier D2D users in the macrocell tier, which is $\left\lfloor 2 N P P_{C}\right\rfloor$.

Furthermore, we assume that small cell base stations (SCBSs) and macrocell users are randomly distributed inside the coverage area of the macrocell. The probability density function (PDF) of its distance $r$ from the macrocell base station (MBS) is

$$
f(r)=\left\{\begin{array}{l}
\frac{2 r}{\left(R_{M}\right)^{2}}\left(r \in\left(R_{\min }, R_{M}-R_{S}\right]\right) \text { for SCBSs } \\
\frac{2 r}{\left(R_{M}\right)^{2}}\left(r \in\left(0, R_{M}\right]\right) \text { for macrocell users }
\end{array}\right.
$$

where $R_{\min }$ is the minimum distance between base stations, and $R_{M}$ and $R_{S}$ are the radius of the macrocell and a small cell, respectively.

For a small cell user, it is randomly and uniformly dropped within the cluster of its small cell, and the PDF of its distance $r$ from its associated SCBS is 


$$
f(r)=\frac{2 r}{\left(R_{S}\right)^{2}}\left(r \in\left(0, R_{S}\right]\right)
$$

In particular, an instance for the distribution of system devices is illustrated in Fig. 2, in which $N=50, M=4, K=16, P=0.4, P_{C}=0.4, R_{M}=0.25 \mathrm{~km}, R_{S}=0.02 \mathrm{~km}$ and $R_{\min }=0.08 \mathrm{~km}$.

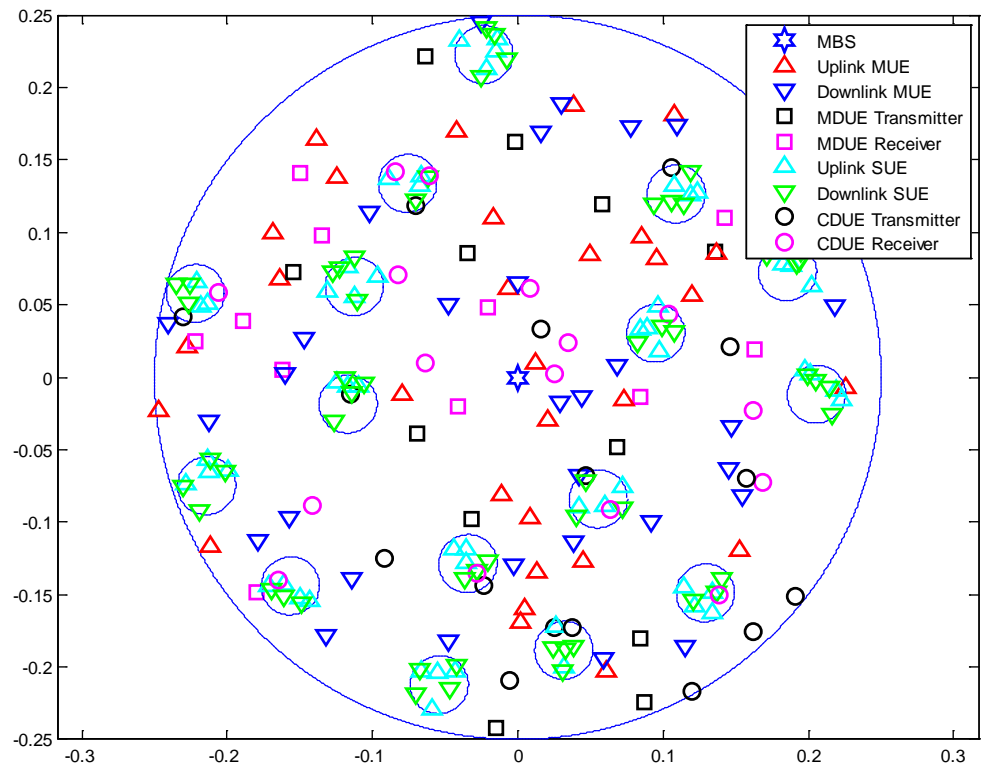

Fig. 2. The distribution of system users in which $N=50, M=4, K=16, P=0.4, P_{C}=0.4, R_{M}=0.25 \mathrm{~km}$, $R_{S}=0.02 \mathrm{~km}$ and $R_{\min }=0.08 \mathrm{~km}$.

\subsection{Communication Modes \& Resource Sharing Strategies}

Because the macro-small cell networks are self-interference systems due to the co-channel deployment between small cells and the macrocell, the interference issue will become more severe to manage after introducing inband D2D communication. To solve this, we devise two resource-sharing strategies, the Cross-Tier Resource Sharing Strategy (CTSS) and Dedicated Resource Sharing Strategy (DS), for D2D users to adapt to the two-tier architecture.

- Cross-Tier Resource Sharing Strategy (CTSS): A D2D pair's transmitter uses the exclusive resource for its own network tier, which can be shared with other tiers' devices. The CTSS does not exploit the full reuse gain, but it simplifies the interference issue by avoiding the interference between D2D users and cellular users in the same tier. This strategy is adopted by MDUE.

- Dedicated Resource Sharing Strategy (DS): A D2D pair uses a dedicated resource in the system, which causes no interference to other devices. This mode is mainly used by CDUE.

The system spectrum sharing strategies are shown in Fig. 3. CDUE $_{M}$ with the DS utilizes the exclusive uplink resource (i.e., the red block), which is not shared by other devices. Moreover, $\mathrm{CDUE}_{\mathrm{S}}$ with the DS utilizes the exclusive downlink resource (i.e., the purple block). Therefore, CDUE causes no interference to other devices. SUE shares the same spectrum with MDUE and MUE. As shown in Fig. 3, the blue spectrum block that is used by MDUE is overlaid with the green one, which is utilized by SUE. Meanwhile, the brown spectrum block utilized by MUE is overlaid with the SUE's as well. Because they share the same resources, SUE and MUE cause interference with each other, and SUE and MDUE interfere with each 
other as well.

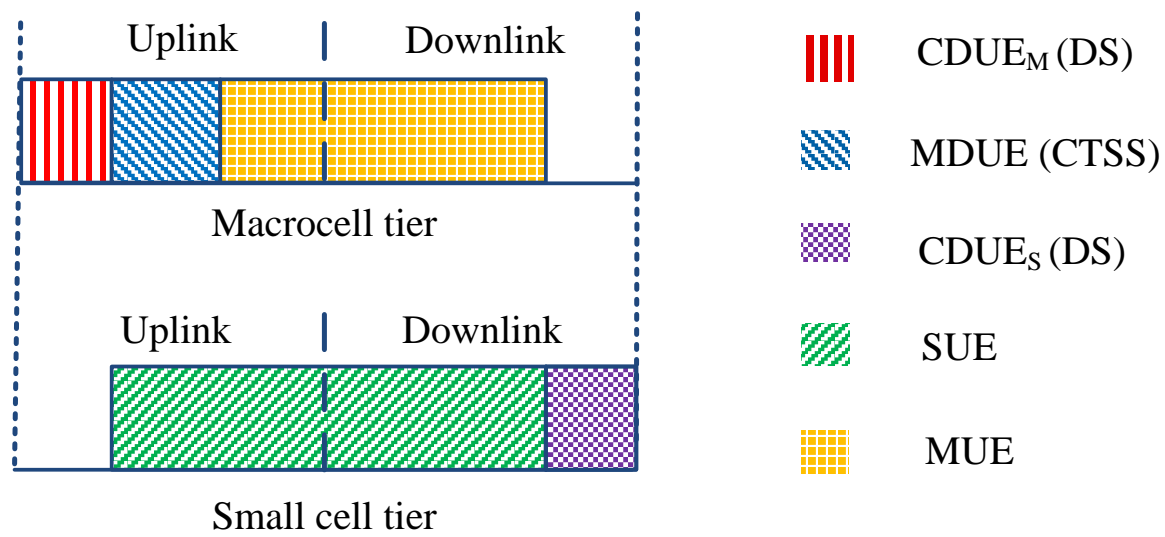

Fig. 3. The spectrum sharing strategies of the system.

\subsection{Achievable Transmission Rate}

We consider the Shannon capacity with outage as the achievable transmission rate between two devices [26]. Let $C_{i j}$ denote the achievable transmission rate between device $i$ and device $j$ :

$$
C_{i j}=\varepsilon \cdot B \log _{2}\left(1+\operatorname{SINR}_{i j}\right)
$$

where $B$ is the bandwidth and $S I N R_{i j}$ is the signal-to-interference plus noise ratio (SINR) of the signal from $i$ to $j . \varepsilon$ is defined as

$$
\varepsilon= \begin{cases}1, & \operatorname{SINR}_{i j} \geq T_{\text {min }} \\ 0, & \operatorname{SINR}_{i j}<T_{\text {min }}\end{cases}
$$

where $T_{\min }$ is the minimum received SINR. If the received SINR is below $T_{\min }$, then the received bits cannot be decoded correctly, and the receiver declares an outage. Therefore, its achievable transmission rate $C_{i j}$ is equal to zero.

We summarize the notation and description of key symbols in Table 1.

Table 1. List of main symbols used in this paper

\begin{tabular}{|c|c|}
\hline Notation & Description \\
\hline \hline$N$ & number of uplink or downlink resource blocks in the entire system \\
\hline$M$ & number of uplink or downlink users in each small cell \\
\hline$P$ & number of small cells in the system \\
\hline$P_{C} / P_{M}$ & probability that D2D communication takes place for each user \\
\hline$R_{S}$ & radius of a small cell \\
\hline$R_{M}$ & radius of a macrocell \\
\hline$R_{\min }$ & fading coefficient in the D2D link \\
\hline$h_{D}$ & fading coefficient in the small cell cellular link \\
\hline$h_{S C}$ & maximum transmit power of D2D users on one resource block \\
\hline$p_{D U E}$ &
\end{tabular}




\begin{tabular}{|c|c|}
\hline$p_{S U E}$ & maximum transmit power of small cell cellular users on one resource block \\
\hline$T_{D}$ & predefined SINR or SNR threshold for D2D direct communication \\
\hline$T_{C}$ & predefined SINR or SNR threshold for cellular communication \\
\hline $\mathbf{S}=\{1,2, \ldots, s\}$ & set of small cell base stations \\
\hline $\mathbf{I}=\{1,2, \ldots, i\}$ & set of MDUE pairs \\
\hline $\mathbf{L}=\{1,2, \ldots, l\}$ & set of users with the cellular mode in the small cells \\
\hline$\alpha_{s, l, i}$ & reuse coefficient, which is a $0-1$ variable \\
\hline$S U E_{l}^{s}$ & l-th user with the cellular mode in the $s$-th small cell \\
\hline$p_{i}^{M D U E}$ & transmit power of the $i$-th MDUE pair \\
\hline$p_{s, l, i}$ & $\begin{aligned} \text { transmit power of the } S U E_{l}^{s} \text { on the resource block that is occupied by both } S U E_{l}^{s} \\
\text { and the } i \text {-th MDUE pair }\end{aligned}$ \\
\hline$S I N R_{i}^{M D U E}$ & received SINR at the $i$-th MDUE pair \\
\hline $\operatorname{SINR}_{\mathrm{s}, l, i}$ & $\begin{array}{l}\text { received SINR at the s-th small cell base station on the resource block occupied by } \\
\text { both } S U E_{l}^{s} \text { and the } i \text {-th MDUE pair }\end{array}$ \\
\hline$d_{C D U E}$ & distance of the CDUE pair \\
\hline$d_{s, l, i}$ & distance between $S U E_{l}^{s}$ and the receiver of the $i$-th MDUE pair \\
\hline$d_{s, l}$ & distance from $S U E_{l}^{s}$ to its associated small cell $s$ \\
\hline$d_{s, i}$ & distance from the transmitter of the $i$-th MDUE pair to small cell $s$ \\
\hline$d_{i}^{M D U E}$ & distance of the $i$-th MDUE pair \\
\hline$C_{i}^{M D U E}$ & achievable transmission rate for the $i$-th MDUE pair in the macrocell tier \\
\hline$C_{s, l, i}$ & $\begin{array}{l}\text { achievable transmission rate for the resource block in the small cell tier, which is } \\
\text { occupied by both } S U E_{l}^{s} \text { and the } i \text {-th MDUE pair }\end{array}$ \\
\hline$\hat{p}_{i, s}^{M D U E}$ & constraint for the $i$-th MDUE pair transmit power of small cell $s$ \\
\hline$F_{i, s}^{M D U E}$ & control factor for the $i$-th MDUE pair of small cell $s$ \\
\hline$I_{i, s}^{M D U E}$ & interference at the receiver of the $i$-th MDUE pair caused by the user in small cell $s$ \\
\hline
\end{tabular}

\section{QoS-aware Interference Management for Cross- and Co-tier D2D}

\subsection{Mode Selection for Cross-tier D2D}

For cross-tier D2D user equipment (CDUE), D2D pairs are encouraged to apply the dedicated resource sharing strategy. When the CDUE pair's transmitter with DS uses the maximum power to transmit a signal $x$, the received signal $y$ at the receiver can be written as

$$
y=h_{D} \sqrt{p_{\text {DUE }}\left(d_{\text {CDUE }}\right)^{-\gamma}} x+n_{0}
$$

where $p_{D U E}$ is the maximum transmit power of D2D users on one RB, $\gamma$ is the path loss exponent, $h_{D}$ stands for the fading coefficient in the D2D link related to the antenna characteristics and the attenuation from blockage [27], $d_{C D U E}$ is the distance of the CDUE pair, and $n_{0}$ denotes the additive white Gaussian noise with zero mean experienced at the CDUE receiver. Let $N_{0}$ denote the noise power. Therefore, the received SNR at the CDUE receiver is given by 


$$
S N R_{C D U E}=\frac{\left|h_{D}\right|^{2} p_{D U E}\left(d_{C D U E}\right)^{-\gamma}}{N_{0}}
$$

To guarantee the QoS of the D2D pair, the received SNR should satisfy $S N R_{C D U E} \geq T_{D}$, where $T_{D}$ is a predefined SINR or SNR threshold for D2D direct communications. If $S N R_{C D E E}$ is lower than $T_{D}$, the communication signal has to be relayed by base stations (i.e., the CDUE pair adopts the cellular mode) because, in this case, the distance of the D2D pair users is too large, and they cannot communicate directly.

\subsection{Joint Power Control and RB Allocation for Co-tier D2D}

For MDUE pairs, if the distance of an MDUE pair is larger than that between the MDUE transmitter and MBS, the MDUE pair will adopt the cellular mode. Otherwise, it is encouraged to apply the CTSS to communicate directly.

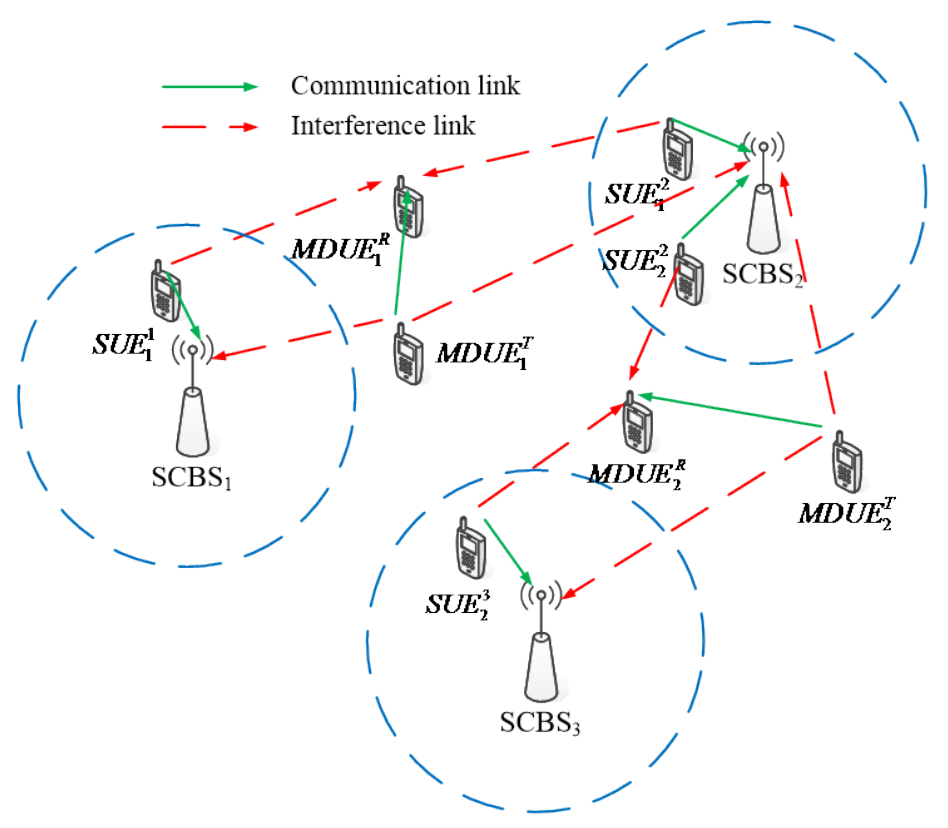

Fig. 4. An illustration for co-tier D2D, where the $M D U E_{1}$ pair uses the same uplink RB with $S U E_{1}^{1}$ and $S U E_{1}^{2}$, and the $M D U E_{2}$ pair reuses the same uplink RB with $S U E_{2}^{3}$ and $S U E_{2}^{2}$.

We use $\mathbf{S}=\{1,2, \ldots, S\}$ to denote the set of small cell base stations and $\mathbf{I}=\{1,2, \ldots, i\}$ to denote the set of MDUE pairs; $M D U E_{i}^{T}$ and $M D U E_{i}^{R}$ are the transmitter and receiver of the $i$-th MDUE pair, respectively. Additionally, let $\mathbf{L}=\{1,2, \ldots, l\}$ denote the set of users with the cellular mode in small cell $s$ (i.e., $S C B S_{s}$ ), and $S U E_{l}^{s}$ stands for the l-th cellular mode user in small cell s. To clarify this, we take an example; as shown in Fig. 4, the $M D U E_{1}$ pair shares the same uplink RB with $S U E_{1}^{1}$ and $S U E_{1}^{2}$. Therefore, $M D U E_{1}^{R}$, which acts as the receiver of the $M D U E_{1}$ pair, suffers interference from $S U E_{1}^{1}$ and $S U E_{1}^{2}$. The $M D U E_{2}$ pair shares the same uplink RB with $S U E_{2}^{2}$ and $S U E_{2}^{3} . M D U E_{2}^{T}$, the transmitter of the $M D U E_{2}$ pair, can 
thus cause interference on $\mathrm{SCBS}_{2}$ and $\mathrm{SCBS}_{3}$. Because the MDUE pair is using the Cross-Tier Resource Sharing Strategy, it will not cause interference with other devices in the macro-tier.

Let $\alpha_{s, l, i}$ denote the resource reuse coefficient, which is a $0-1$ variable. If $\alpha_{s, l, i}=1, S U E_{l}^{s}$ reuses the same uplink RB occupied by the $M D U E_{i}$ pair. Otherwise, $\alpha_{s, l, i}=0$. When $M D U E_{i}^{T}$ transmits a signal $x_{i}$ to $M D U E_{i}^{R}$ with CTSS, and $S U E_{l}^{s}$ transmits a signal $x_{l, s}$ to its base station $S C B S_{S}$, the received signal at $M D U E_{i}^{R}$ can be written as

$$
y_{i}=h_{D} \sqrt{p_{i}^{M D U E}\left(d_{i}^{M D U E}\right)^{-\gamma}} x_{i}+\sum_{s \in \mathrm{S}} \sum_{l \in \mathbf{L}}\left(\alpha_{s, l, i} h_{D} \sqrt{p_{s, l, i}\left(d_{s, l, i}\right)^{-\gamma}} x_{l, s}\right)+n_{0}
$$

where $p_{i}^{M D U E}$ and $d_{i}^{M D U E}$ respectively represent the transmit power and the distance of the $M D U E_{i}$ pair. $p_{s, l, i}$ denotes the transmit power of the $S U E_{l}^{s}$ on the RB that is shared by the $M D U E_{i}$ pair and $S U E_{l}^{s}$. The distance between $S U E_{l}^{s}$ and $M D U E_{i}^{R}$ is denoted by $d_{s, l, i}$. Because each individual MDUE pair can only use one RB while one SUE can use multiple RBs, there may be interference between several MDUE pairs and one SUE .

Based on equation (7), the received SINR at $M D U E_{i}^{R}$ is given by

$$
\operatorname{SINR}_{i}^{M D U E}=\frac{\left|h_{D}\right|^{2} p_{i}^{M D U E} /\left(d_{i}^{M D U E}\right)^{-\gamma}}{N_{0}+\sum_{s \in \mathbf{S}} \sum_{l \in \mathbf{L}}\left(\alpha_{s, l, i}\left|h_{D}\right|^{2} p_{s, l, i} /\left(d_{s, l, i}\right)^{-\gamma}\right)}
$$

Similarly, the SINR at the $S C B S_{s}$ on the RB occupied by both $S U E_{l}^{s}$ and the $M D U E_{i}$ pair is given by

$$
\operatorname{SINR}_{s, l, i}=\frac{\alpha_{s, l, i}\left|h_{S C}\right|^{2} p_{s, l, i} /\left(d_{s, l}\right)^{-\gamma}}{N_{0}+\left|h_{S C}\right|^{2} p_{i}^{M D U E} /\left(d_{s, i}\right)^{-\gamma}}
$$

where $h_{S C}$ is the fading coefficient of the small cell cellular link and $d_{s, l}$ and $d_{s, i}$ are the distances from $S C B S_{s}$ to $S U E_{l}^{s}$ and $M D U E_{i}^{R}$, respectively. We ignore the co-tier interference from the other small cells because we set a minimum separation for base stations.

To optimize the sum outage capacity of MDUE pairs and small cell uplink cellular users, the optimization problem can be formulated as:

$$
\max _{\boldsymbol{a}, \mathbf{Q}}\left(\sum_{i \in \mathbf{I}} C_{i}^{M D U E}+\sum_{s \in \mathbf{S}} \sum_{l \in \mathbf{L}} \sum_{i \in \mathbf{I}} C_{s, l, i}\right)
$$

where $\boldsymbol{\alpha}=\left\{\alpha_{s, l, i}\right\}_{i \in \mathbf{I}, l \in \mathbf{L}, s \in \mathbf{S}}, \mathbf{Q}=\left\{p_{i}^{M D U E}, p_{s, l, i}\right\}_{i \in \mathbf{I}, l \in \mathbf{L}, s \in \mathbf{S}}, C_{i}^{M D U E}$ is the achievable transmission rate for the $M D U E_{i}$ pair in the macrocell tier, and $C_{s, l, i}$ is the achievable transmission rate for the RB in the small cell tier, which is shared by $S U E_{l}^{s}$ and the $M D U E_{i}$ pair.

Based on equations (3) and (4), equation (10) can be rewritten as:

$$
\max _{\boldsymbol{a}, \mathbf{Q}}\left[\sum_{i \in \mathbf{I}} \log _{2}\left(1+\operatorname{SINR} R_{i}^{M D U E}\right)+\sum_{s \in \mathbf{S}} \sum_{l \in \mathbf{L}} \sum_{i \in \mathbf{I}} \log _{2}\left(1+\operatorname{SINR}_{s, l, i}\right)\right]
$$

s.t. 


$$
\begin{aligned}
& \operatorname{SINR}_{i}^{M D U E} \geq T_{D}, \forall i \in \mathbf{I} \\
& \operatorname{SINR}_{s, l, i} \geq T_{C}, \alpha_{s, l, i}=1, \forall i \in \mathbf{I}, l \in \mathbf{L}, s \in \mathbf{S} \\
& p_{i}^{M D U E} \leq p_{D U E}, \forall i \in \mathbf{I} \\
& p_{s, l, i} \leq p_{S U E}, \forall i \in \mathbf{I}, l \in \mathbf{L}, s \in \mathbf{S} \\
& \sum_{l \in \mathbf{L}} \alpha_{s, l, i} \leq 1, \forall i \in \mathbf{I}, s \in \mathbf{S} \\
& \alpha_{s, l, i} \in\{0,1\}, \forall i \in \mathbf{I}, l \in \mathbf{L}, s \in \mathbf{S}
\end{aligned}
$$

where $p_{\text {SUE }}$ is the maximum transmit power of a small cell cellular user on one RB and $T_{C}$ is a system predefined value that is adopted to maintain the cellular link quality. Equation (12) guarantees the QoS of the $M D U E_{i}$ pair. When $\alpha_{s, l, i}=1, S U E_{l}^{s}$ reuses the RB occupied by the $M D U E_{i}$ pair, equation (13) guarantees the QoS of $S U E_{l}^{s}$. The transmit power limitations on one RB of a D2D user and cellular mode user are given in equations (14) and (15). Equation (16) guarantees the orthogonal use of RBs for the cellular mode users in each small cell.

The optimization problem (11) is a non-convex optimization due to the uncertainty of interference terms in the instantaneous SINR in equations (8) and (9), which is caused by the varying of reuse coefficients. Therefore, it is a challenging task to obtain the optimal solution directly. Alternatively, we propose a suboptimal and efficient solution in the following section.

\section{Heuristic Algorithm for Co-tier D2D to solve the Optimization Problem}

In this section, we design a heuristic algorithm for co-tier D2D users to find a suboptimal solution for the non-convex combinatorial optimization problem as shown in equation (11). This algorithm is a joint method with power control and RB allocation, which aims to mitigate the interference and reduce the computational complexity when solving the problem in equation (11). The procedure of the proposed algorithm is presented in Algorithm 1.

For the sake of resource reuse gain and the improvement of spectrum efficiency, in initialization, for each RB occupied by the $M D U E_{i}$ pair ( $i \in \mathbf{I}$ ), a small cell $s$ randomly chooses a cellular user $l$ (i.e., $S U E_{l}^{s}$ ) to reuse it, i.e., $\alpha_{s, l, i}=1$, and sets the transmit power of $S U E_{l}^{s}$ on the RB, which is occupied by both the $M D U E_{i}$ pair and $S U E_{l}^{s}$, to the maximum value (i.e., $p_{s, l, i}=p_{S U E}$ ).

To enable cellular communication, equation (13) has to be satisfied. Assume that the channel state information (CSI) can be collected by measurements and/or feedback from the user equipment [28], small cell $s$ calculates the maximum transmit power for $M D U E_{i}^{T}$ as follows:

$$
p_{i, s}^{M D U E}=\left\{\begin{array}{l}
\frac{\left(d_{s, i}\right)^{-\gamma}}{\left|h_{S C}\right|^{2}}\left(\frac{\alpha_{s, l, i}\left|h_{S C}\right|^{2} p_{S U E}}{T_{C}\left(d_{s, l, i}\right)^{-\gamma}}-N_{0}\right), \alpha_{s, l, i}=1 \\
p_{D U E}, \alpha_{s, l, i}=0
\end{array}\right.
$$

The transmit power constraint for $M D U E_{i}^{T}$ can be given as: 


$$
\hat{p}_{i, s}^{M D U E}=\min \left\{p_{i, s}^{M D U E}, p_{D U E}\right\}
$$

For a small cell $s, s \in \mathbf{S}$, there is an exclusive constraint for the $M D U E_{i}$ pair's transmit power, as shown in equation (19). Let $\hat{\mathbf{P}}_{i, s}^{M D U E}=\left\{\hat{p}_{i, 1}^{M D U E}, \hat{p}_{i, 2}^{M D U E}, \ldots, \hat{p}_{i, s}^{M D U E}\right\}$ denote the set of constraints; then, we set the transmit power of the $M D U E_{i}$ pair $p_{i}^{M D U E}=\min \left(\hat{\mathbf{P}}_{i, s}^{M D U E}\right)$.

Based on equation (8), the macrocell BS can obtain the $S I N R_{i}^{M D U E}$. If the $\operatorname{SINR}_{i}^{M D U E}$ does not satisfy equation (12), the receiver of the $M D U E_{i}$ pair (i.e., $M D U E_{i}^{R}$ ) suffers severe interference from the small cell tier and requires further action, e.g., the design of an RB allocation strategy for interference mitigation.

To this end, we devise a control factor denoted as $F_{i, s}^{M D U E}$ and let $F_{i, s}^{M D U E}=I_{i, s}^{M D U E}-\hat{p}_{i, s}^{M D U E}$ for the small cell $s$ and the $M D U E_{i}$ pair, where $I_{i, s}^{M D U E}$ is the interference at $M D U E_{i}^{R}$ caused by the user in the small cell $s$. $I_{i, s}^{\text {MDUE }}$ can be obtained as

$$
I_{i, s}^{M D U E}=\sum_{l \in \mathbf{L}}\left(\alpha_{s, l, i}\left|h_{D}\right|^{2} p_{S U E} /\left(d_{s, l, i}\right)^{-\gamma}\right)
$$

Let $\mathbf{F}_{i, s}^{M D U E}=\left\{F_{i, 1}^{M D U E}, F_{i, 2}^{M D U E}, \ldots, F_{i, s}^{M D U E}\right\}$ denote the set of control factors. MBS first chooses the small cell that has the maximum control factor, then sets $\alpha_{s, l, i}=0$ and updates $\hat{\mathbf{P}}_{i, s}^{M D U E}$ and $p_{i}^{M D U E}$ until equation (12) is satisfied. These selected small cells, for which $\alpha_{s, l, i}$ equals zero, are usually too close to $M D U E_{i}^{R}$ or $M D U E_{i}^{T}$, and it is inappropriate to reuse the RB, which is occupied by $M D U E_{i}$. The computational complexity of the proposed heuristic algorithm is $O\left(K\left\lfloor N P P_{M}\right\rfloor\right)$, where $\mathrm{K}$ is the number of small cells in the system and $\left\lfloor N P P_{M}\right\rfloor$ is the number of MDUE pairs.

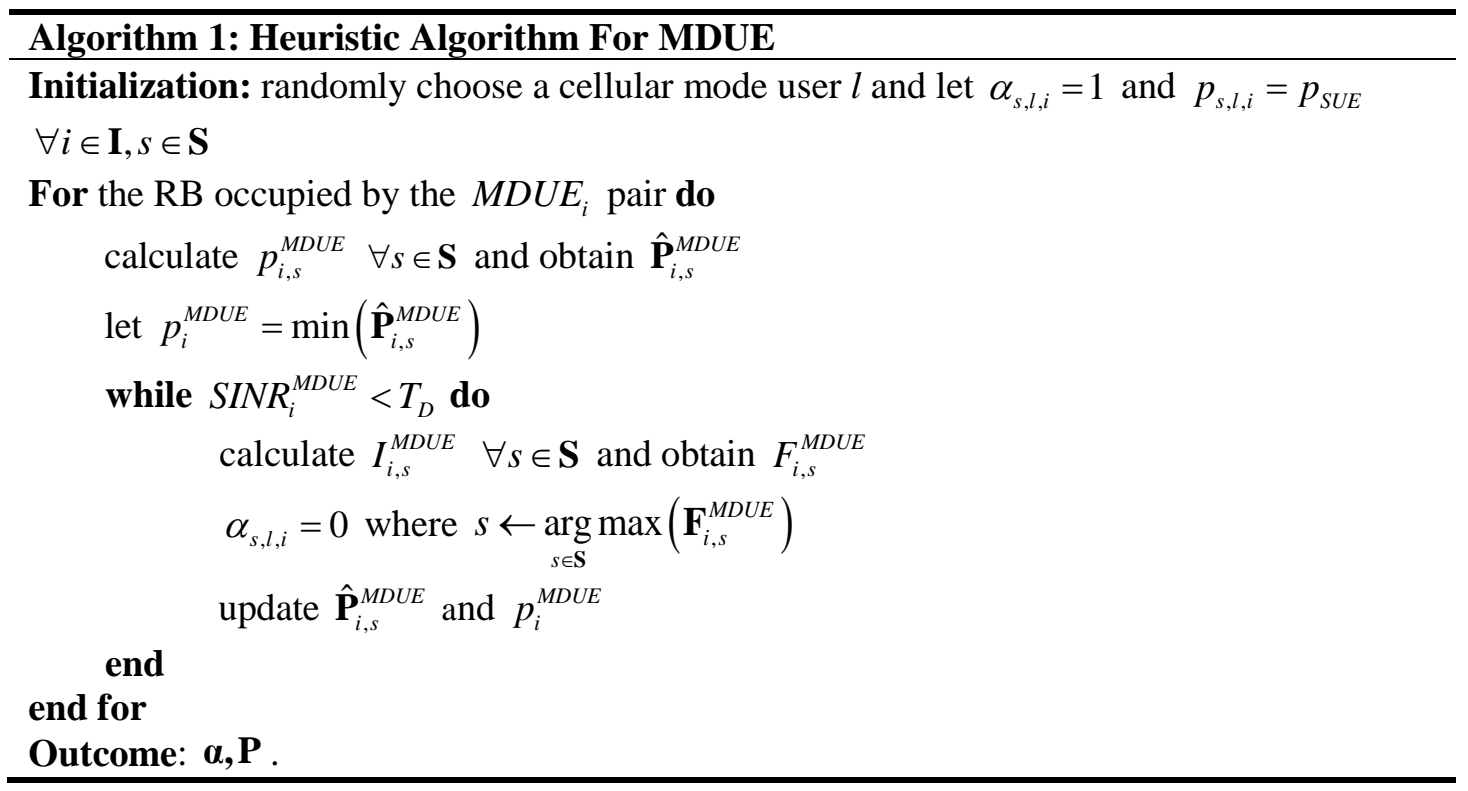




\section{Numerical Results}

In this section, we evaluate the performance of co- and cross-tier D2D underlaying macro-small cell networks. We consider a two-tier network with a set of small cells located in a macrocell. The performance measure is computed after averaging 1000 independent runs. The key simulation parameters are set up according to [7] [29] and are given in Table 2.

Table 2. Key simulation parameters

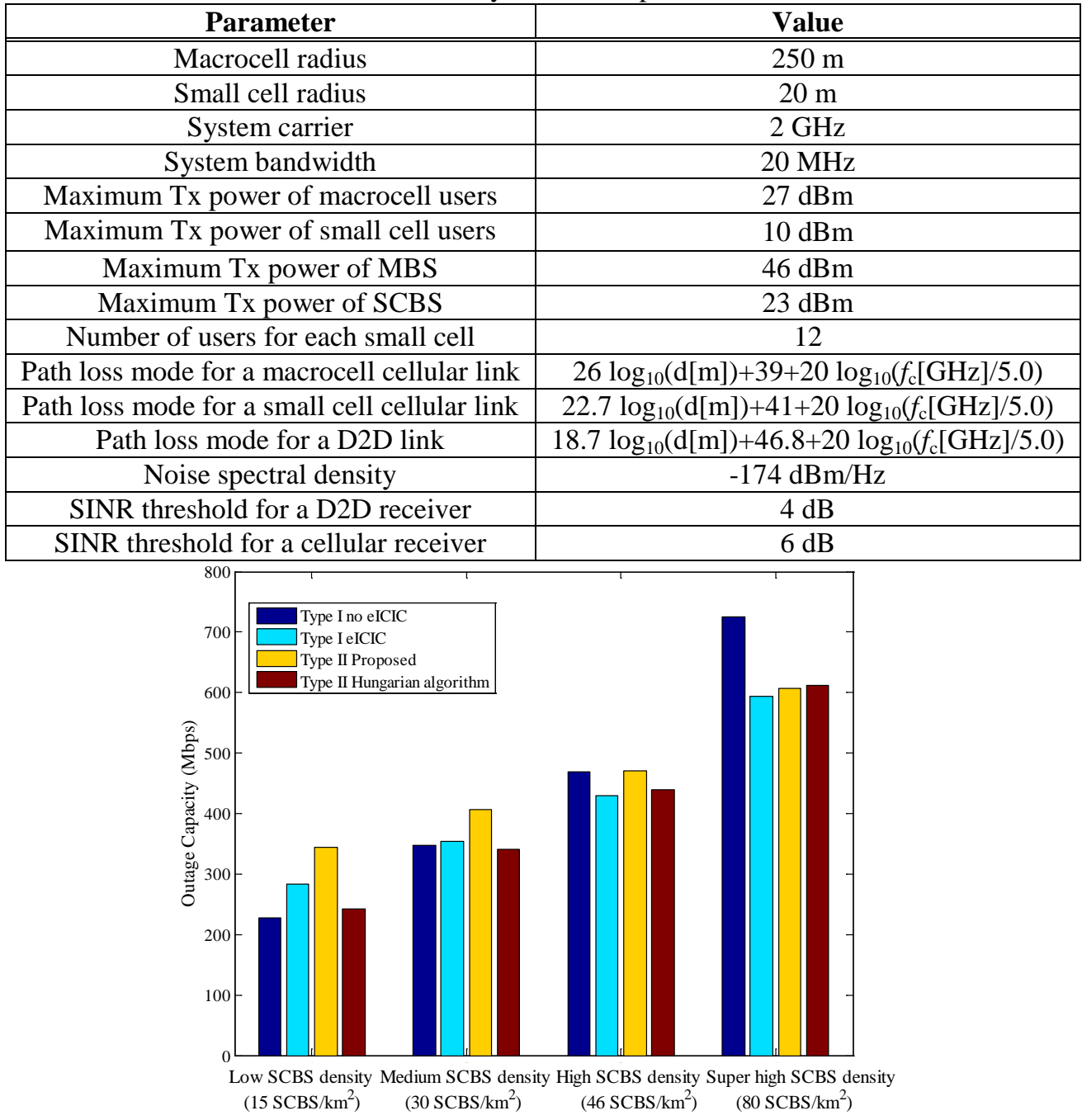

Fig. 5. Overall capacity under four cases with various SCBS densities in which $P=0.6$ and $P_{M}=0.8$.

We first look into the capacity of the two-tier network enabling the co-tier and cross-tier D2D communications. Fig. 5 depicts the overall capacity under four cases with various SCBS densities. We use 'Type I' to denote the macro-small cell networks that are without the D2D direct communication, that is, all D2D users are forced to use the cellular mode (CM). 'Type II' denotes the D2D heterogeneous networks that allow D2D users to perform mode selection and adopt the direct communication mode. Moreover, we use 'no eICIC' to denote small cells that are without any enhanced inter-cell interference coordination (eICIC) and 'eICIC' to denote small cells that apply the fourth power control approach (objective SINR of MUE) listed in [30], 
which is to guarantee a minimum SINR at the MUEs while controlling the maximum transmit power of small cell base stations. 'Hungarian algorithm' means that MDUE pairs conduct the RB allocation with the Hungarian algorithm and the power control schemes proposed in [16].

In Fig. 5, it is observed that 'Type II Proposed,' which stands for the Type II D2D heterogeneous network with the proposed D2D interference management algorithm, achieves the best capacity performance in low and medium SCBS densities. This is because, with the CDUE pairs adopting the DS to communicate directly, the cross-tier interference from the CDUE pairs is avoided; moreover, the MDUE pairs with the CTSS communicating directly also mitigate the cross-tier interference because the transmit power of MDUE is much smaller than MBS. In high and super-high SCBS densities, the overall capacity of the 'no eICIC' case is higher than that of 'eICIC.' That is because taking no action at the small cells (i.e., 'no eICIC') results in worse macrocell user protection but better small cell throughput performance compared with the 'eICIC' cases. The small cell throughput takes almost all of the system capacity for high and super-high SCBS densities.

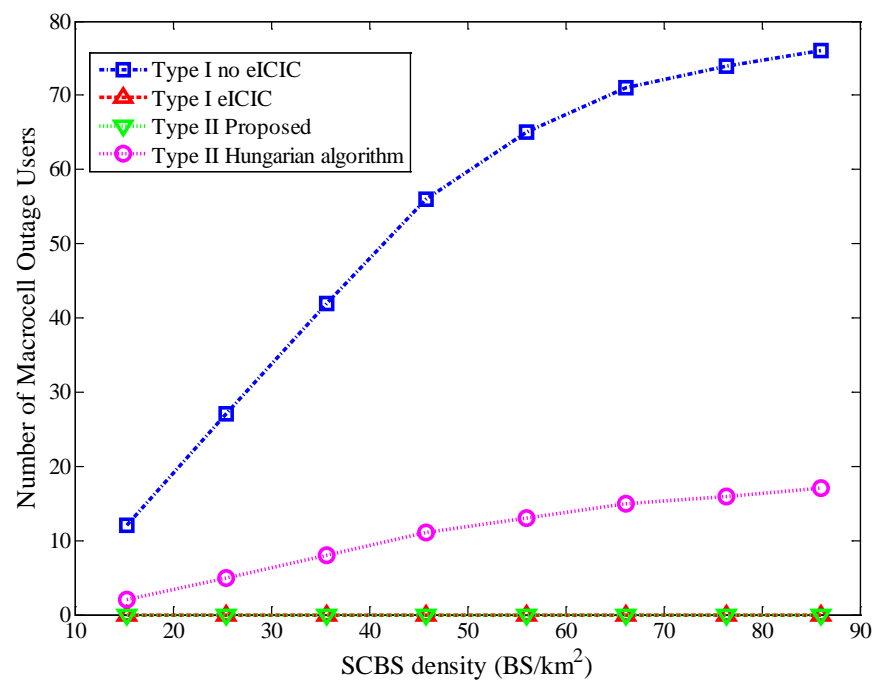

Fig. 6. The number of macrocell outage users under four cases with various SCBS densities in which $P=0.6$ and $P_{M}=0.8$.

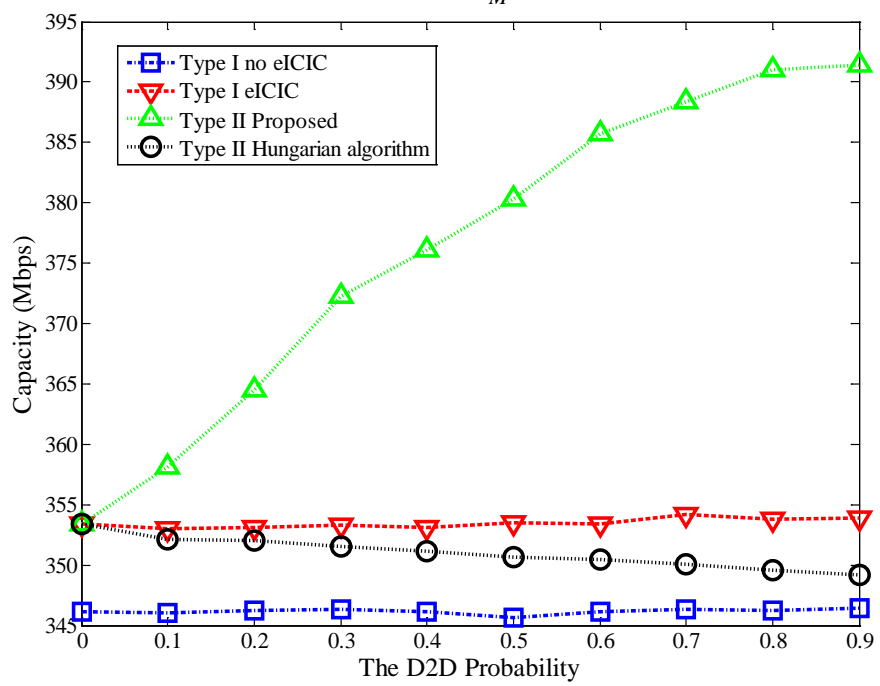

Fig. 7. Overall capacity versus the D2D probability in which $P_{\mathrm{C}}=0.4, P_{M}=0.6$ and $30 \mathrm{SCBS} / \mathrm{km}^{2}$ 
We then assess the QoS of macrocell users. Fig. 6 depicts the number of macrocell outage users with various SCBS densities. We can see that the 'eICIC' and 'proposed' interference management algorithms have better performance in avoiding the outage of users. With the increase of the SCBS density, the numbers of macrocell outage users of 'Type I no eICIC' and 'Type II Hungarian algorithm' rise stably. These two cases sacrifice the QoS of macrocell users, including cellular users and D2D users, in trade for better small cell throughput performance, which is also supported in Fig. 5.

Fig. 7 illustrates the overall capacity versus the D2D probability. With the increase of the D2D probability, the overall capacity of 'Type II Proposed' grows significantly. That is because there are more CDUE pairs and MDUE pairs to avoid and mitigate the cross-tier interference, respectively, and improve the capacity performance with the growth of the D2D probability.

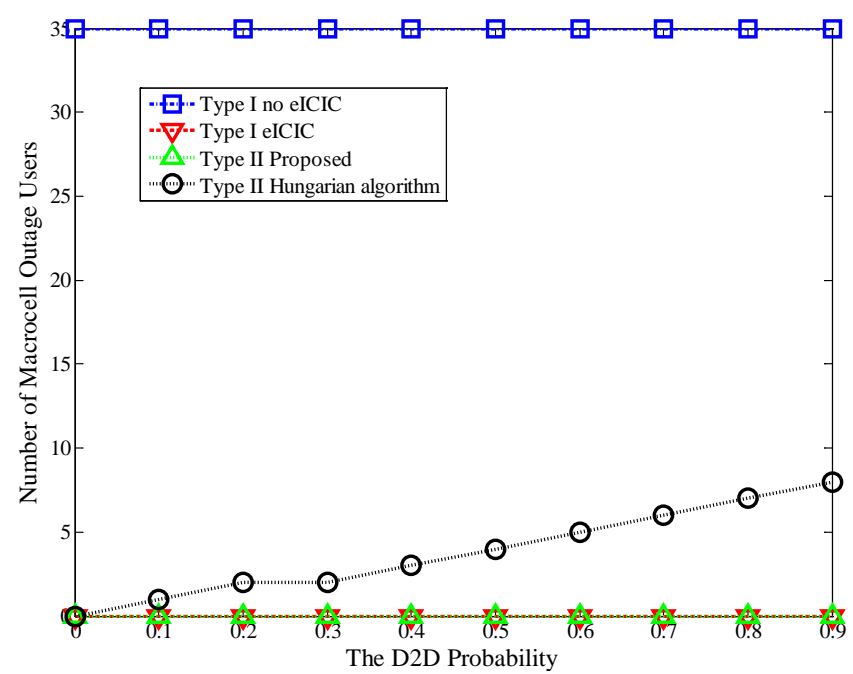

Fig. 8. The number of macrocell outage users versus the D2D probability with $P_{C}=0.4$ and $P_{M}=0.6$.

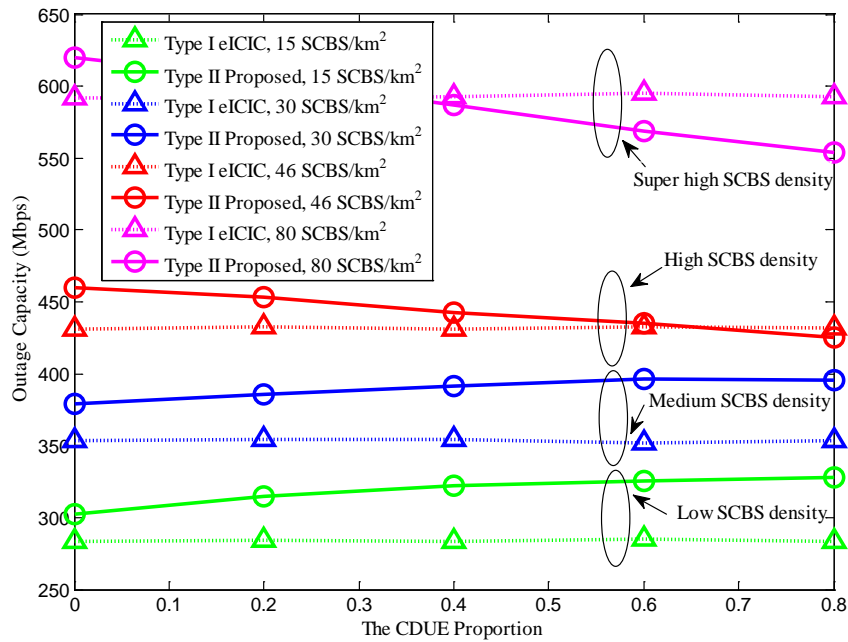

Fig. 9. Overall capacity with various CDUE proportions and SCBS densities in which $P=0.4$. 
Fig. 8 depicts the number of macrocell outage users versus the D2D probability. It is observed that the number of macrocell outage users in the 'Type II Hungarian algorithm' case increases gradually with the growth of the D2D probability, and it also leads to the decline of overall capacity, as shown in Fig. 7. The reason is that, with the growth of the D2D probability, there are more and more MDUE in the system, and the inter-channel interference between MDUE and small cell users becomes more severe. While the Hungarian algorithm cannot manage the interference very well, the extravagant interference leads to an increase of the number of MDUE outages.

We also look into how the overall capacity changes with various CDUE proportions and SCBS densities, as shown in Fig. 9, while $P=0.4$. The dashed lines in Fig. 9 represent the outage capacity with various SCBS densities when the macro-small cell networks are without D2D communication, i.e., all D2D users are using the cellular mode. Therefore, the dashed lines are approximately invariant during the increase of the CDUE proportion for various cases of SCBS density. The solid lines describe the outage capacity of 'Type II Proposed' with the varying of the CDUE proportion and density of small cells. With the rise of the CDUE proportion, the overall outage capacity grows stably for low and medium SCBS densities but declines for higher SCBS densities. The reason behind this is that the CDUE pairs with the Dedicated Resource Sharing Strategy (DS) can avoid interference and yield benefits for macro-small cell networks. Alternately, more RBs need to be assigned to the CDUE pairs with the increase of the CDUE proportion. As a result, the number of available RBs for the small cell tier is decreasing. Therefore, when the SCBS density is low or medium, the growth of the outage capacity is attributed to the benefits of the CDUE pairs. When the SCBS density is high, the outage capacity declines due to insufficient RBs for dense small cell deployment.

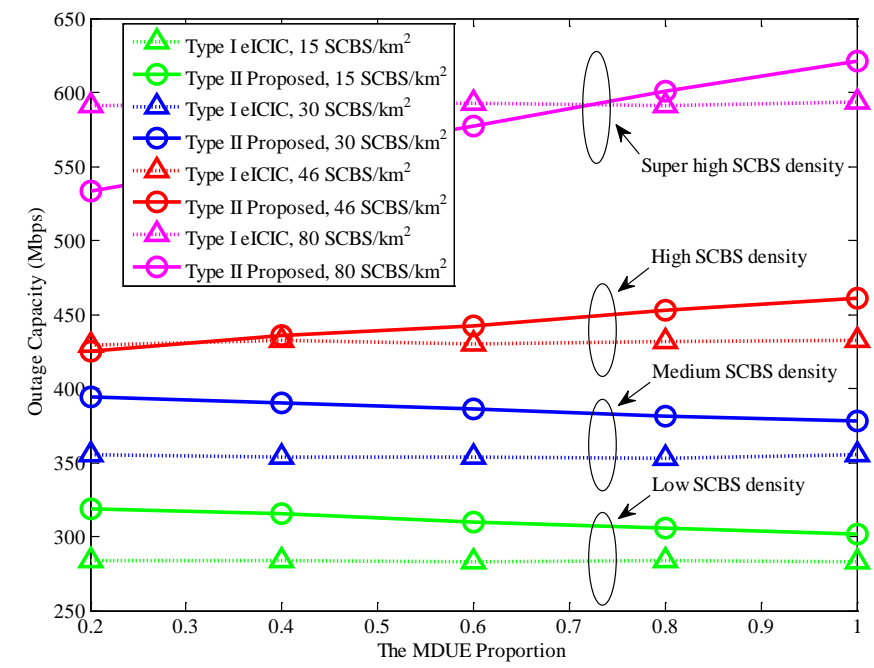

Fig. 10. Overall capacity with various MDUE proportions and SCBS densities when $P=0.6$.

Furthermore, we study the impact of the MDUE proportion on system capacity. Fig. 10 describes the overall capacity with various MDUE proportions and SCBS densities when $P=0.6$. In low and medium SCBS densities, the outage capacity of 'Type II proposed,' shown via the solid lines, declines with the growth of the MDUE proportion. In other words, the CDUE pairs yield more benefits than MDUE pairs, which is also supported in Fig. 9. In addition, when all 
D2D pairs are MDUE, i.e., the MDUE proportion is equal to one, similar to the situation in which the CDUE proportion is equal to zero in Fig. 9, the macro-small cell system with D2D communication can obtain better performance than traditional macro-small cell networks with various cases of SCBS density. This reveals that the co-tier D2D can obtain steady benefits for macro-small cell systems during the densification of small cells.

\section{Conclusions}

In this paper, we have investigated the performance of cross- and co-tier D2D communications underlaying two tier macro-small cell networks. We divide the system users into four types: MUE, MDUE, SUE and CDUE. Then, we propose two resource-sharing strategies, CTSS and DS for co-tier and cross-tier D2D, respectively, to avoid the interference between D2D users and cellular users in the same tier. For co-tier D2D, we formulate the joint optimization problem of power control and resource reuse with the purpose of maximizing the overall outage capacity. Because the joint optimization problem is non-convex, we devise a heuristic algorithm to obtain a suboptimal solution and guarantee the QoS requirements of all users.

The system-level simulations indicate that the cross-tier D2D pairs adopting DS and the cross-tier D2D pairs with the CTSS can avoid and mitigate the interference, respectively, and yield benefits for macro-small cell networks. The simulations also reveal that cross-tier D2D can obtain better performance for low and medium SCBS densities but lower performance for high SCBS density. Moreover, the co-tier D2D can achieve a steady improvement with the increase of SCBS density. In conclusion, our study presents the high potential of both cross-tier D2D and co-tier D2D for the future deployment of heterogeneous small cell networks.

\section{Acknowledgment}

This work was supported in part by the National Natural Science Foundations of China (No. 61301148 and No. 61272061), the Fundamental Research Funds for the Central Universities of China, Hunan Natural Science Foundation of China.

\section{References}

[1] M. Jo, T. Maksymyuk and B. Strykhalyuk, et al, "Device-to-Device based heterogeneous radio access network architecture for mobile cloud computing,” IEEE Wireless Communications, vol. 22, no. 3, pp. 50-58, June, 2015. Article (CrossRef Link)

[2] F. Boccardi, R. W. Heath, A. Lozano, et al, "Five disruptive technology directions for 5G," IEEE Communications Magazine, vol. 52, no. 2, pp. 74-80, February, 2014. Article (CrossRef Link)

[3] J. Liu, N. Kato, J. Ma, et al, "Device-to-Device communication in LTE-Advanced networks: a survey,” IEEE Communications Surveys \& Tutorials, vol. 17, no. 4, pp. 1923-1940, December, 2014. Article (CrossRef Link)

[4] J. G. Andrews, S. Buzzi, W. Choi, et al, "What will 5G be?” IEEE Journal on Selected Areas in Communications, vol. 32, no. 6, pp. 1065-1082, June, 2014. Article (CrossRef Link)

[5] S. M. Corson, R. Laroia, J. Li, et al. “Toward proximity-aware internetworking," IEEE Wireless Communications, vol. 17, no. 6, pp. 26-33, December, 2010. Article (CrossRef Link)

[6] 3GPP TR 22.803 v12.2.0, “Feasibility study for proximity services (ProSe).” 2013. Article (CrossRef Link)

[7] 3GPP TR 36.843 v12.0.1, "Study on LTE Device-to-Device Proximity Services Radio Aspects." 2014. Article (CrossRef Link) 
[8] H. ElSawy, E. Hossain, M. -S. Alouini, “Analytical Modeling of Mode Selection and Power Control for Underlay D2D Communication in Cellular Networks," IEEE Transactions on Communications, vol. 62, no. 11, pp. 4147-4161, November, 2014. Article (CrossRef Link)

[9] C. H. Yu, K. Doppler, C. B. Ribeiro, et al, "Resource sharing optimization for Device-to-Device communication underlaying cellular networks,” IEEE Transactions on Wireless Communications, vol. 19, no. 8, pp. 2752-2763, August, 2011. Article (CrossRef Link)

[10] A. Asadi, Q. Wang, V. Mancuso, "A survey on Device-to-Device communication in cellular networks,” IEEE Communications Surveys \& Tutorials, vol. 16, no. 4, pp. 1801-1819, April, 2014. Article (CrossRef Link)

[11] S. Mumtaz, J. Rodriguez, Smart device to smart device communication, Springer International Publishing, 2014. Article (CrossRef Link)

[12] S. Y. Kim, C. H. Lim, C. H. Cho, "Performance analysis of a dense Device to Device network," KSII Transactions on Internet and Information Systems (TIIS), vol. 8, no. 9, pp. 2967-2981, September, 2014. Article (CrossRef Link)

[13] J. Wang, D. Zhu, C. Zhao, et al, "Resource sharing of underlaying Device-to-Device and uplink cellular communications,” IEEE Communications Letters, vol. 17, no. 6, pp. 1148-1151, June, 2013. Article (CrossRef Link)

[14] J. Niu, W. Guo, "Resource allocation for cooperative relay based wireless D2D networks with selfish users,” KSII Transactions on Internet \& Information Systems (TIIS), vol. 9, no. 6, June, 2015. Article (CrossRef Link)

[15] H. Wang, X. Chu, "Distance-constrained resource-sharing criteria for Device-to-Device communications underlaying cellular networks,” Electronics letters, vol. 48, no. 9, pp. 528-530, April, 2012. Article (CrossRef Link)

[16] Y. Xu, R. Yin, T. Han, et al, "Interference-aware channel allocation for Device-to-Device communication underlaying cellular networks," in Proc. of 1st IEEE International Conference on Communications in China (ICCC), pp. 422-425, August 15-17, 2012. Article (CrossRef Link)

[17] W. Xu, L. Liang, H. Zhang, et al, "Performance enhanced transmission in Device-to-Device communications: Beamforming or interference cancellation?” in Proc. of 2012 IEEE Global Communications Conference (GLOBECOM), pp. 4296-4301, December 3-7, 2012. Article (CrossRef Link)

[18] C. X. Wang, F. Haider and X. Gao, et al, "Cellular architecture and key technologies for 5G wireless communication networks,” IEEE Communications Magazine, vol. 52, no. 2, pp. 122-130, February, 2014. Article (CrossRef Link)

[19] M. Jo, T. Maksymyuk, R. L. Batista, et al, “A survey of converging solutions for heterogeneous mobile networks,” IEEE Wireless Communications, vol. 21, no. 6, pp. 54-62, December, 2014. Article (CrossRef Link)

[20] K. Zheng, Y. Li, Y. Zhang, et al, "Performance analysis and evaluation of deployment in small cell networks,” KSII Transactions on Internet and Information Systems (TIIS), vol. 9, no. 3, pp. 886-900, March, 2015. Article (CrossRef Link)

[21] Hossain E, Rasti M, Tabassum H, et al, "Evolution toward 5G multi-tier cellular wireless networks: An interference management perspective,” IEEE Wireless Communications, vol. 21, no. 3, pp. 118-127, June, 2014. Article (CrossRef Link)

[22] L. Wei, R. Hu, Y. Qian, et al, "Enable Device-to-Device communications underlaying cellular networks: challenges and research aspects,” IEEE Communications Magazine, vol. 52, no. 6, pp. 90-96, June, 2014. Article (CrossRef Link)

[23] Z. Shangwei, L. Jiajia, K. Nei, et al, “Average Rate Analysis for a D2D Overlaying Two-Tier Downlink Cellular Network,” in Proc of 2015 IEEE International Conference on Communications, pp. 3376-3381, June 8-12, 2015. Article (CrossRef Link)

[24] H. Monowar, H. Ekram, "Distributed resource allocation in D2D-enabled multi-tier cellular networks: an auction approach," in Proc of 2015 IEEE International Conference on Communications, pp. 2949-2954, June 8-12, 2015. Article (CrossRef Link)

[25] J. Hoydis, M. Kobayashi, M. Debbah, “Green small-cell networks,” IEEE Vehicular Technology Magazine, vol. 6, no. 1, pp. 37-43, March, 2011. Article (CrossRef Link) 
[26] A. Goldsmith, Wireless Communications, Cambridge University Press, 2013. Article (CrossRef Link)

[27] T. Rappaport, Wireless Communications: Principles and Practice (Second Edition), Prentice-Hall PTR, 2002. Article (CrossRef Link)

[28] C. H. Yu, K. Doppler, C. Ribeiro, et al, "Performance impact of fading interference to device-to-device communication underlaying cellular networks." in Proc. of 2009 IEEE 20th International Symposium on Personal, Indoor and Mobile Radio Communications, pp. 858-862, September 13-16, 2009. Article (CrossRef Link)

[29] WINNER II D1.1.2, “WINNER II Channel Models,” September, 2007. Article (CrossRef Link)

[30] D. Lopez-Perez, S. Güvenç, G. De la Roche, et al, "Enhanced intercell interference coordination challenges in heterogeneous networks," IEEE Wireless Communications, vol. 18, no. 3, pp. 22-30, June, 2011. Article (CrossRef Link)
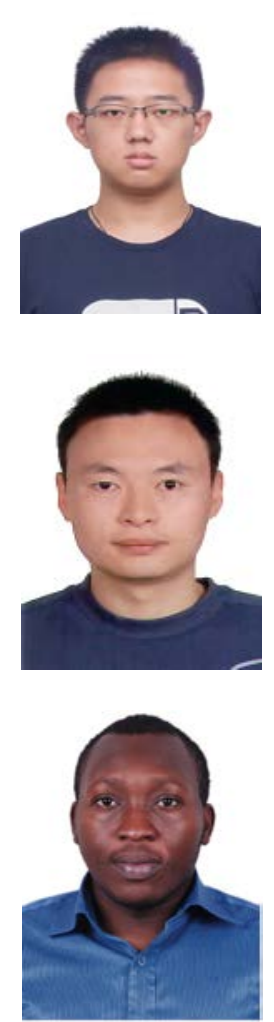

Tong $\mathbf{L i}$ received the B.S. degree in communication engineering from Hunan University, China, in 2014. He is currently pursuing the M.S. degree in communication and information system at Hunan University, His interests focus on power and resource management in future wireless and cellular networks.

Zhu Xiao received his M.S.degree in communication and information system from Xidian University, China, in 2007. He joined the State Key Laboratory of Integrated Services Networks (ISN) at Xidian University in 2007 and obtained his Ph.D degree in Dec. 2009. Since 2010, he has been a lecturer with College of Computer Science and Electronic Engineering, Hunan University, China. From May 2010 to February 2012, He was a research fellow in Department of Computer Science and Technology, University of Bedfordshire, UK. His research interests include wireless/mobile communications, wireless localization, Next-generation communications and heterogeneous networks.

Hassana Maigary Georges received a B.Tech from N'gaoundéré University, Cameroon, in 2007 and a M.Eng.C.S.Tech from Hunan University in 2011. He is currently pursuing a Ph.D. degree in the College of Computer Science and Electronic Engineering at Hunan University. His research interests includewireless communications, cooperative localization, signal processing, detection and estimation for navigation systems. 
Li et al.: Performance Analysis of Co- and Cross-tier Device-to-Device Communication Underlaying Macro-small Cell Wireless Networks

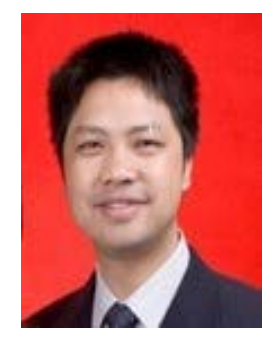

Zhinian Luo received his Ph.D degree of communication and information systems from Shanghai Jiaotong University in 2009. He served in the department of mobile radio communication of Zhongxing Comm. Inc. developing UMTS Node B in 1999. From 2001 to 2003, he jointed Alcatel Co. as a hardware engineer in TND (Transmission Network Department). He is currently an associate professor in the college of computer science and electronic engineering of Hunan University. His research interests include mobile radio channel modeling, multi-user detection, channel estimation and advanced signal processing for next generation wireless communication systems.

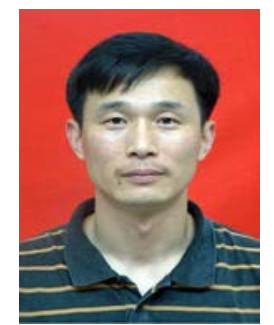

Dong Wang received the B.S. degree and Ph.D. degree in computer science from Hunan University in 1986 and 2006, respectively. From December 2004 to December 2005, he was a visiting scholarship in University of technology, Sydney, Australia. Since 1986, he has been working with Hunan University, China. Currently, he is professor of Hunan University. His main research interests include network test and performance evaluation, wireless communications and mobile computing. 